\title{
FAIXAS NORMAIS DE NUTRIENTES EM CANA-DE-AÇÚCAR PELOS MÉTODOS ChM, DRIS E CND E NÍVEL CRÍTICO PELA DISTRIBUIÇÃO NORMAL REDUZIDA ${ }^{(1)}$
}

\author{
Elcio Ferreira dos Santos ${ }^{(2)}$, Riviane Maria Albuquerque Donha ${ }^{(2)}$, Cecília Miranda Magno \\ de Araújo ${ }^{(3)}$, José Lavres Junior ${ }^{(4)}$ \& Marcos Antonio Camacho ${ }^{(5)}$
}

\begin{abstract}
RESUMO
Existem diversos métodos para a interpretação da diagnose foliar; o nível crítico ou as faixas de suficiência descritas na literatura são os mais utilizados. Porém, o uso de métodos de determinação de faixas normais de nutrientes que contemplam regiões específicas (localidades) aumenta a eficiência da interpretação. $O$ objetivo deste trabalho foi determinar as faixas normais de nutrientes para a cultura da cana-de-açúcar, mediante a utilização dos métodos Chance Matemática (ChM), Sistema Integrado de Diagnose e Recomendação (DRIS) e Diagnose da Composição Nutricional (CND), além do Nível Crítico determinado pelo método de distribuição normal reduzida. $O$ trabalho foi realizado tomando-se como base dados relativos a teores de nutrientes em amostras foliares e de produtividade de lavouras comerciais de cana-de-açúcar, localizadas no município de Campos de Goytacazes, RJ. Com o método da ChM, foram obtidas faixas de suficiência semelhantes aos métodos DRIS e CND, para $\mathrm{N}$, Ca, S e Mn, enquanto para $\mathrm{P}, \mathrm{K}, \mathrm{Mg}$, $\mathrm{Cu}$ e $\mathrm{Zn}$ os valores obtidos pela $\mathrm{ChM}$ foram superiores. A utilização dos métodos ChM, DRIS e CND, em lavouras comerciais de cana-de-açúcar em geral, possibilitou a obtenção de menor amplitude da faixa normal dos nutrientes, em comparação com os valores alcançados pelos métodos nível crítico e faixa de suficiência.
\end{abstract}

Termos de indexação: Saccharum officinarum, diagnose foliar, estado nutricional.

(1) Recebido para publicação em 12 de setembro de 2012 e aprovado em 22 de julho de 2013.

(2) Mestrando(a) em Energia Nuclear na Agricultura e no Ambiente, Centro de Energia Nuclear na Agricultura - CENA/USP. Av. Centenário, 303. Caixa Postal 96. CEP 13416-000 Piracicaba (SP). E-mail: elciosantos@cena.usp.br, mdonha@cena.usp.br

(3) Mestranda, Instituto Nacional de Pesquisa da Amazônia - INPA. Av. André Araújo, 2936. CEP: 69060-001 Manaus (AM). Email: ceciliaagro@gmail.com

(4) Professor, Laboratório de Nutrição Mineral de Plantas - CENA/USP. E-mail: jlavres@cena.usp.br

(5) Professor, Universidade Estadual de Mato Grosso do Sul, Campus de Aquidauana. Rodovia Aquidauana-Piraputanga, km 12. Caixa Postal 25. CEP 79200-000 Aquidauana (MS). E-mail: camacho@uems.br 


\title{
SUMMARY: NORMAL NUTRIENT RANGES FOR SUGARCANE BY THE METHODS ChM, DRIS AND CND AND CRITICAL LEVEL BY REDUCED NORMAL DISTRIBUTION
}

\begin{abstract}
There are several interpretation methods for foliar nutrient analysis, and the critical level or nutrient sufficient range described in the literature are the most commonly used. However, an inclusion of methods that determine the normal nutrient ranges for specific regions (sites) can increase the efficiency of interpretation. The objective of this study was to compare normal nutrient ranges for sugarcane cultivation, determined by the methods ChM (Mathematical Chance), DRIS (Integrated System of Diagnosis and Recommendation) and CND (Compositional Nutrient Diagnosis), and the Critical Level, by the method of reduced normal distribution. The study was carried out based on data of foliar nutrient contents and crop yield, from commercial sugarcane fields in Campos de Goytacazes, State of Rio de Janeiro. By the ChM method, sufficiency ranges for $N, C a, S$, and $M n$ were similar to those determined by DRIS and CND, while for P, K, Mg, Cu, and $\mathrm{Zn}$ the values obtained by ChM were higher. In general, the use of ChM, DRIS and CND in commercial sugarcane fields reduced the amplitude of the normal nutrient ranges, compared with the values obtained by the critical level and sufficiency range methods.
\end{abstract}

Index terms: Saccharum officinarum, leaf analysis, nutritional status.

\section{INTRODUÇÃO}

A cana-de-açúcar (Saccharum officinarum L.) foi introduzida no Brasil no período colonial e transformou-se em uma das principais culturas do agronegócio brasileiro. O Brasil é o maior produtor mundial de cana-de-açúcar, sendo responsável por mais da metade do açúcar comercializado no mundo. A estimativa da área plantada com cana-de-açúcar, na safra 2012/2013, é de 8,6 milhões de ha e com produção estimada de, aproximadamente, 600 milhões de toneladas (CONAB, 2012). Além disso, compromissos internacionais para utilização de fontes renováveis de energia e redução das emissões de gases do efeito estufa deverão promover nos próximos anos, no Brasil, aumento considerável da área plantada com cana-de-açúcar, visando a produção de etanol.

As variedades utilizadas, as condições climáticas e o manejo e a correção da fertilidade do solo influenciam na composição química das plantas. A resposta a esses fatores pode ser determinada por meio da diagnose foliar que se constitui em alternativa eficiente para a avaliação nutricional das culturas, como a cana-de-açúcar (Reis Junior \& Monerat, 2002).

Existem diversos métodos de interpretação da diagnose foliar. O nível crítico ou as faixas de suficiência descritas na literatura são as mais utilizadas para essa interpretação. Todavia, a utilização de métodos que possam determinar valores de referência para diagnose nutricional regionalizados pode proporcionar maior acurácia quanto aos métodos utilizados na diagnose do estado nutricional das plantas, sem a necessidade de ensaios de calibração e com resultados promissores (Camacho et al., 2012a). Assim, como métodos alternativos, destacam-se Chance Matemática (ChM), Sistema Integrado de Diagnose e Recomendação (DRIS) e Diagnose de
Composição Nutricional (CND), bem como o Nível Crítico pelo método de distribuição normal reduzida.

A ChM é um processo matemático de cálculo de probabilidades e interpretação de grande volume de dados nutricionais, que indica a faixa de valores de um dado fator correlacionado à planta, em que se espera identificar a máxima produtividade. Por meio de esse método obtêm-se as faixas infraótima, ótima e supraótima, os níveis crítico e ótimo, classificando as áreas analisadas segundo sua distribuição em cada uma das faixas de suficiência (Wadt et al., 1998).

O DRIS corresponde aos valores médios das relações entre os nutrientes de uma população não anormal ou população de referência (Beaufils, 1973), admitindo-se que as relações entre dois nutrientes são melhores indicadores do estado nutricional das plantas, calculando-se um índice para cada nutriente e considerando a sua relação com os demais (Mourão Filho et al., 2002). Os índices DRIS positivos indicam excesso; os DRIS de valores negativos, deficiência, enquanto os DRIS iguais, ou próximos a zero, equilíbrio nutricional (Wadt et al., 2012).

O CND assemelha-se ao DRIS principalmente pelo fato de ordenar a limitação dos nutrientes na planta (Parent \& Dafir, 1992) e possuem as vantagens de trabalhar os nutrientes numa mesma unidade, além de possui melhor fundamentação estatística. Parent et al. (1994) indicaram que a análise multivariada do CND fornece meios para definir uma subpopulação de alto rendimento. A abordagem CND tem uma robusta base matemática para definir uma meta de rendimento mínimo útil para discriminar entre o alto e baixo rendimento das subpopulações.

A utilização dos métodos ChM, DRIS e CND para determinar faixas normais de nutrientes tem sido empregada para diversas culturas como a soja (Glycine max) (Urano et al., 2007), o algodoeiro 
(Gossypium hirsutum r. latifolium) (Serra et al., 2010; Camacho et al., 2012b) e a laranjeira-pera (Citrus sinensis) (Camacho et al., 2012a), com resultados promissores para a calibração de teores ótimos a partir de dados provenientes de monitoramento nutricional de lavouras comerciais.

Pesquisas que visem à interpretação de resultados nutricionais por diferentes métodos para a cultura da cana-de-açúcar fazem-se necessárias e poderão fornecer informações acerca da ciclagem de nutrientes nos canaviais (Otto et al., 2010). Tais informações servem de suporte para a agroindústria sucroalcooleira, que poderá introduzir métodos de adubação mais eficientes, com menores desperdícios e economicamente mais viáveis.

Objetivaram-se com este trabalho determinar e comparar as faixas normais de nutrientes para a cultura da cana-de-açúcar, utilizando os métodos Chance Matemática (ChM), Sistema Integrado de Diagnose e Recomendação (DRIS), Diagnose da Composição Nutricional (CND) e Nível Crítico, pelo método de distribuição normal reduzida, com base em dados de uma população constituída por lavouras comercias de cana-de-açúcar.

\section{MATERIAL E MÉTODOS}

Este estudo foi desenvolvido utilizando-se amostras de uma população de plantas de lavouras comerciais de cana-planta e cana-soca (var. CB45-3, RB72-454, RB73-9735 e SP70-1143), na região do município de Campos dos Goytacazes, RJ. Os dados de produtividade acumulada são referentes aos anos 1996 e 1997. Cada amostra foi constituída por 15 folhas da posição +3 , coletadas de plantas com quatro meses de desenvolvimento (Malavolta et al., 1997), aleatoriamente nas áreas de cultivo de, aproximadamente, 1 ha. As folhas amostradas foram utilizadas para análise química, considerando-se apenas os $20 \mathrm{~cm}$ medianos e descartando-se a nervura central. Os dados de produtividade foram registrados nos locais amostrados quando a cana-de-açúcar atingiu o ponto de colheita.

Foram utilizados para as análises os dados dos teores totais de nutrientes de 50 amostras foliares e a produtividade da cana-de-açúcar oriunda de talhões com média de $60 \mathrm{t} \mathrm{ha}^{-1}$. Para o ajuste do método, a população foi dividida em duas classes: uma com produtividade acima de $78 \mathrm{t} \mathrm{ha}^{-1}$, definida como subpopulação de alta produtividade, cuja produção de cana foi superior à média adicionada a 0,5 desvio-padrão; e outra com produtividade abaixo dessa.

A análise pelo método da ChM foi realizada, conforme indicações de Wadt et al. (1998). Para cada nutriente em estudo, os teores foliares foram classificados em ordem crescente e distribuídos em um número de classes definido como a raiz quadrada do número de observações.

Os intervalos de valores de cada classe foram então determinados, dividindo-se a amplitude dos teores do nutriente em questão pelo número de classes estabelecido, de acordo com Wadt et al. (1998). Em cada classe, calculou-se a chance matemática $\left(\mathrm{ChM}_{\mathrm{i}}\right)$, conforme a equação:

$$
\operatorname{ChM}_{\mathrm{i}}=\left[\operatorname{ChM}\left(\mathrm{A}_{\mathrm{i}} / \mathrm{A}\right) \times \operatorname{ChM}\left(\mathrm{A}_{\mathrm{i}} / \mathrm{C}_{\mathrm{i}}\right)\right]^{0,5}
$$

em que $\mathrm{ChM}(\mathrm{Ai} / \mathrm{A})=\mathrm{P}(\mathrm{Ai} / \mathrm{A}) \times \mathrm{PRODi} ; \mathrm{P}(\mathrm{Ai} / \mathrm{A})=$ frequência de lavouras de alta produtividade na classe i, em relação ao total geral de talhões de alta produtividade; $\mathrm{PRODi}=$ produtividade média dos talhões de alta produtividade na classe i $\left(\mathrm{t} \mathrm{ha}^{-1}\right)$; $\mathrm{ChM}(\mathrm{Ai} / \mathrm{Ci})=\mathrm{P}(\mathrm{Ai} / \mathrm{Ci})$ x PRODi e $\mathrm{P}(\mathrm{Ai} / \mathrm{Ci})=$ frequência de talhões de alta produtividade na classe i, em relação ao total geral de talhões na classe i. Os limites inferiores e superiores das classes de teor de nutrientes que apresentaram as maiores chances matemáticas foram considerados a faixa normal.

Para a determinação das faixas normais pelo método DRIS, foram calculados a média, o coeficiente de variação e a variância para o grupo de referência e de baixa produtividade, utilizando todas as relações possíveis (ex.: A/B ou B/A). Para cada par de nutrientes, a forma de expressão do quociente entre eles que forneceu a maior razão de variância entre o grupo de baixa produtividade e de referência foi selecionada para ser usada no DRIS, conforme descrito por Walworth et al. (1986) e Hartz et al. (1998). As funções intermediárias para geração dos índices DRIS foram calculadas pela fórmula de Beaufils (1971), simplificada por Jones (1981):

$$
\mathrm{f}(\mathrm{A} / \mathrm{B})=(\mathrm{A} / \mathrm{B}-\mathrm{a} / \mathrm{b}) \mathrm{K} / \mathrm{s}
$$

em que $\mathrm{A} / \mathrm{B}=$ valor da relação entre as concentrações dos dois nutrientes na planta sob diagnóstico; $\mathrm{a} / \mathrm{b}$ e $\mathrm{s}=$ as médias e os desvios-padrão das relações (A/B) entre nutrientes das amostras da população de referência; e $\mathrm{K}=10$ (constante de valor arbitrário).

Como valores nulos dos índices DRIS caracterizam condição de equilíbrio nutricional, uma vez que os valores dos índices refletem os desvios padronizados sem relação aos valores de referência, podem-se estimar os teores ótimos de nutrientes por meio do ajuste de modelos estatísticos da relação entre teores foliares de nutrientes e os índices DRIS, na subpopulação de alta produtividade. A faixa normal foi obtida pela definição dos teores correspondentes ao intervalo de índices DRIS entre - 2/3 s e 2/3 s (Wadt et al., 1998; Urano et al., 2007).

As normas CND foram constituídas pela média aritmética e pelo desvio-padrão das variáveis multinutrientes na população de alta produtividade, de acordo com Khiari et al. (2001). As variáveis multinutrientes (VA) consistiram do logaritmo neperiano do quociente entre a concentração de cada nutriente (A) $\left(\mathrm{g} \mathrm{kg}^{-1}\right)$ e a média geométrica das concentrações dos constituintes da matéria seca $(G)$, adaptado de Khiari et al. (2001): 


$$
\begin{gathered}
\mathrm{G}=(\mathrm{A} \times \mathrm{B} \times \ldots \times \mathrm{C} \times \mathrm{R})^{1 / \mathrm{n}-1} \\
\mathrm{VA}=\ln (\mathrm{A} / \mathrm{G})
\end{gathered}
$$

Os índices CND foram calculados pela diferença entre as variáveis multinutrientes no talhão avaliado (VA) e a média da população de referência (VA*), dividida pelo desvio-padrão dessa variável na população de referência $\left(\mathrm{SA}^{*}\right)$ :

$$
\mathrm{IA}=\left(\mathrm{VA}-\mathrm{VA}^{*}\right) / \mathrm{SA}^{*}
$$

O limite inferior e o superior da faixa normal de concentração de nutrientes pelo método CND foram determinados de modo análogo ao utilizado pelo método DRIS (Urano et al., 2007).

A obtenção do nível crítico pela distribuição contínua de probabilidade, proposta por Maia et al. (2001), é com base na distribuição normal reduzida. Para isso, utilizam-se os dados de produtividade $(\mathrm{P})$ e de $\mathrm{Q}$, em que $\mathrm{Q}$ é definido como a relação entre $\mathrm{P}$ e ni $(\mathrm{Q}=\mathrm{P} / \mathrm{ni})$, sendo ni o teor do nutriente para o qual se deseja encontrar o nível crítico. O nível crítico é obtido pela fórmula:

$$
\mathrm{NC}_{\mathrm{i}}=\left(1,281552 \mathrm{~s}_{1}+\mathrm{w}_{1}\right) /\left(1,281552 \mathrm{~s}_{2}+\mathrm{w}_{2}\right)
$$

em que $\mathrm{w}_{1}$ e $\mathrm{s}_{1}$ são a média aritmética e o desviopadrão de $\mathrm{P} ; \mathrm{e}_{2}$ e $\mathrm{s}_{2}$, a média e o desvio-padrão de $\mathrm{Q}$, respectivamente. A pressuposição básica para se encontrar o nível crítico pela distribuição contínua de probabilidade é a de que os dados de $\mathrm{P}$ e $\mathrm{Q}$ sigam distribuição normal. Para isso, testou-se a normalidade das variáveis citadas por meio do teste de Lilliefors (Conover, 1971). Quando não se observou a normalidade dos dados, esses foram transformados pelo logaritmo neperiano (ln).

\section{RESULTADOS E DISCUSSÃO}

Os maiores valores para $\mathrm{N}$ foram obtidos pelo método da ChM na classe $3\left(13,0\right.$ - 15, $0 \mathrm{~g} \mathrm{~kg}^{-1}$ ) (Quadro 1). Esses resultados foram semelhantes aos obtidos pelo método DRIS (12,6 - 15,2 $\left.\mathrm{g} \mathrm{kg}^{-1}\right)$ e CND (12,6 - 16,5 $\left.\mathrm{g} \mathrm{kg}^{-1}\right)$, porém estão abaixo do relatado na literatura (Raij et al., 1996; Malavolta et al., 1997). Esses resultados diferem dos encontrados por Serra et al. (2010), os quais observaram que as faixas adequadas para o teor de N, obtidas por meio dos valores da ChM, DRIS e CND, foram semelhantes às das recomendações oficiais existentes por meio de nível crítico. (Quadro 1).

Para $\mathrm{P}$, os maiores valores de $\mathrm{ChM}$ foram obtidos nos limites da classe 6 , correspondendo à faixa de 2,5 a $2,8 \mathrm{~g} \mathrm{~kg}^{-1}$ (Quadro 1), muito embora a classe 6 apresente a maior concentração de talhões com alta produtividade dentro dos intervalos para o $\mathrm{P}$. Também a faixa normal encontrada pelo método $\mathrm{ChM}$ foi superior aos índices DRIS (1,8 - 2,3 $\left.\mathrm{g} \mathrm{kg}^{-1}\right)$ e CND $\left(1,5-2,2 \mathrm{~g} \mathrm{~kg}^{-1}\right)$ e aos teores adequados relatados por Raij et al. (1996) (Quadro 2), embora assemelhem-se aos resultados propostos por Malavolta et al. (1997).
A determinação da faixa normal quanto ao teor de K para produção de $78 \mathrm{t} \mathrm{ha}^{-1}$ ficou entre $12,7 \mathrm{e}$ $14,3 \mathrm{~g} \mathrm{~kg}^{-1}$ (Quadro 1). A faixa normal está em concordância com o encontrado na literatura (Raij et al., 1996; Malavolta et al., 1997), porém acima dos índices DRIS (9,6 - 12,5 g kg-1) e CND (7,6 - 12,0 $\left.\mathrm{g} \mathrm{kg}^{-1}\right)$, encontrados neste trabalho (Quadro 2).

A faixa normal para $\mathrm{Mg}$ associada a produtividades superiores a $78 \mathrm{t} \mathrm{ha}^{-1}$ foi de 3,7 - 4,3 $\mathrm{g} \mathrm{kg}^{-1}$ (Quadro 1), superior ao encontrado na literatura, bem como pelos índices DRIS e CND desta pesquisa (Quadro 2).

A faixa normal para Ca obtida na classe 2 (Quadro 1) foi idêntica aos índices DRIS (2,6 - 3,6 $\left.\mathrm{g} \mathrm{kg}^{-1}\right)$ (Quadro 2). Para S, a faixa de suficiência foi determinada pela classe 5 , que apresentou maior $\mathrm{ChM}$ para produtividade, sendo os limites $\left(1,9-2,3 \mathrm{~g} \mathrm{~kg}^{-1}\right)$ dessa classe definidos como a faixa normal para os teores de S (Quadro 1). As faixas normais para Ca e S obtidas pelos métodos ChM, DRIS e CND foram menores às descritas por Raij et al. (1996) e Malavolta et al. (1997) (Quadro 2).

Da mesma forma com que se determinaram os valores de ChM para macronutrientes, estimou-se de modo probabilístico a chance de classes de teores para alguns micronutrientes $(\mathrm{Cu}, \mathrm{Mn}$ e $\mathrm{Zn})$ apresentarem produtividade superior a 78 t ha $^{-1}$ (Quadro 3).

$\mathrm{Na}$ determinação das faixas normais para $\mathrm{Cu}$, observou-se maior valor de ChM na classe 2 , com limites de suficiência entre 4,6 e 6,6 $\mathrm{mg} \mathrm{kg}^{-1}$ (Quadro 3). Essa faixa normal, por sua vez, foi maior que os índices DRIS (3,8 - 5,0 $\left.\mathrm{mg} \mathrm{kg}^{-1}\right)$ e CND (3,8 - 5,6 $\left.\mathrm{mg} \mathrm{kg}^{-1}\right)$. Contudo, os três métodos apresentaram faixa normal inferior ao descrito na literatura (Quadro 2).

A faixa normal para Mn obtida na classe $3(56,7$ - 73,0 $\mathrm{mg} \mathrm{kg}^{-1}$ ) foi semelhante aos valores relativos aos índices DRIS e CND (Quadro 2). As faixas normais quantificadas pelos três métodos estiveram dentro da amplitude da faixa encontrada por Malavolta et al. (1997) e Raij et al. (1996).

Os maiores valores de ChM para $\mathrm{Zn}$ foram obtidos no intervalo da classe 7 (Quadro 2), sendo a faixa normal apresentada pelo método ChM (24,8 - 27,9 $\mathrm{mg} \mathrm{kg}^{-1}$ ) superior à faixa normal obtida pelos métodos DRIS (12,6 - 14,8 $\left.\mathrm{mg} \mathrm{kg}^{-1}\right)$ e CND (13,0 - $16,4 \mathrm{mg} \mathrm{kg}^{-1}$ ). As faixas de suficiência encontradas na literatura (Quadro 2) apresentaram limite superior muito maior do que o encontrado pelos métodos ChM, DRIS e CND neste trabalho.

Os três métodos apresentaram faixas normais para micronutrientes com amplitude menor, quando comparadas às da literatura (Quadro 2). Resultados semelhantes foram observados por Serra et al. (2010), os quais, comparando as faixas normais dos métodos ChM, DRIS e CND em algodão, obtiveram menor amplitude.

As faixas ótimas para macros e micronutrientes foram comparadas aos níveis críticos propostos por Maia et al. (2001) (Quadro 2). Os níveis críticos 
discriminados pelo critério de distribuição normal reduzida estiveram sempre dentro das faixas ótimas, determinadas pelos métodos DRIS e CND, exceto para os índices DRIS, relativos ao Ca e Mn (Quadro 2). A faixa normal para $\mathrm{K}, \mathrm{Ca}, \mathrm{Mg}, \mathrm{S}, \mathrm{Mn}$ e $\mathrm{Zn}$, definida pelo método ChM, apresentou intervalo de valores não correspondente ao nível crítico.

Foram ajustadas equações de regressão para relacionar teores de nutrientes em folhas de cana-de- açúcar e índices DRIS. Os dados foram ajustados seguindo modelos lineares $(p<0,01)$. Os coeficientes de determinação $\left(R^{2}\right)$ para macro e micronutrientes (Quadro 4) estiveram entre 0,53, para as relações de $\mathrm{Ne} \mathrm{S}$ nas folhas e os índices DRIS desses nutrientes, e 0,83, para os teores de Mn nas folhas e os índices DRIS desse micronutriente.

Em relação à determinação da faixa normal de nutrientes pelo método $\mathrm{CND}$, foram definidos modelos

Quadro 1. Valores de chance matemática $(\mathrm{ChM})$ (1) estabelecidos para diferentes classes de distribuição de teores de macronutrientes em amostras de folhas de cana-de-açúcar coletadas em uma lavoura comercial, em 1996-1997

\begin{tabular}{|c|c|c|c|c|c|c|c|}
\hline Classe & Nutriente & $\mathrm{LIi}^{(2)}$ & $\mathbf{L S i}^{(3)}$ & $\mathbf{P} 1^{(4)}$ & $\mathrm{P} 2^{(5)}$ & PRODi $^{(6)}$ & ChM \\
\hline & & \multicolumn{2}{|c|}{$-\mathrm{g} \mathrm{kg}^{-1}$} & & & $\%$ & $\mathrm{tha}^{-1}$ \\
\hline 1 & $\mathrm{~N}$ & 8,99 & 11,01 & 0 & 15 & 53,72 & 0 \\
\hline 2 & & 11,01 & 13,02 & 3 & 15 & 63,24 & 12,64 \\
\hline 3 & & 13,00 & 15,00 & 7 & 44 & 58,89 & 16,04 \\
\hline 4 & & 15,04 & 17,06 & 2 & 32 & 55,94 & 5,10 \\
\hline 5 & & 17,06 & 19,07 & 3 & 16 & 72,59 & 14,05 \\
\hline 1 & $\mathrm{P}$ & 1,12 & 1,40 & 0 & 22 & 42,49 & 0 \\
\hline 2 & & 1,40 & 1,68 & 2 & 32 & 55,88 & 5,10 \\
\hline 3 & & 1,68 & 1,96 & 4 & 24 & 62,30 & 13,13 \\
\hline 4 & & 1,96 & 2,23 & 4 & 27 & 66,44 & 13,20 \\
\hline 5 & & 2,23 & 2,51 & 2 & 13 & 67,08 & 9,60 \\
\hline 6 & & 2,50 & 2,80 & 3 & 6 & 88,28 & 27,91 \\
\hline 7 & & 2,79 & 3,07 & 0 & 2 & 48,43 & 0 \\
\hline 1 & $\mathrm{~K}$ & 5,05 & 6,59 & 0 & 12 & 41,96 & 0 \\
\hline 2 & & 6,59 & 8,13 & 3 & 24 & 54,88 & 8,67 \\
\hline 3 & & 8,13 & 9,67 & 6 & 43 & 63,40 & 14,97 \\
\hline 4 & & 9,67 & 11,22 & 3 & 34 & 60,52 & 8,04 \\
\hline 5 & & 11,22 & 12,76 & 1 & 10 & 67,73 & 5,53 \\
\hline 6 & & 12,76 & 14,30 & 2 & 3 & 91,05 & 27,14 \\
\hline 1 & $\mathrm{Ca}$ & 1,65 & 2,64 & 3 & 22 & 60,54 & 9,99 \\
\hline 2 & & 2,64 & 3,63 & 8 & 34 & 59,85 & 21,20 \\
\hline 3 & & 3,63 & 4,63 & 4 & 35 & 58,22 & 10,16 \\
\hline 4 & & 4,63 & 5,62 & 0 & 16 & 59,40 & 0 \\
\hline 5 & & 5,62 & 6,61 & 0 & 10 & 58,80 & 0 \\
\hline 1 & $\mathrm{Mg}$ & 0,94 & 1,50 & 0 & 12 & 41,96 & 0 \\
\hline 2 & & 1,50 & 2,06 & 3 & 24 & 54,88 & 8,67 \\
\hline 3 & & 2,06 & 2,62 & 6 & 43 & 63,40 & 14,97 \\
\hline 4 & & 2,62 & 3,17 & 3 & 34 & 60,52 & 8,04 \\
\hline 5 & & 3,17 & 3,73 & 1 & 10 & 67,73 & 5,53 \\
\hline 6 & & 3,73 & 4,29 & 2 & 3 & 91,05 & 27,14 \\
\hline 1 & $\mathrm{~S}$ & 0,32 & 0,46 & 0 & 1 & 55,57 & 0 \\
\hline 2 & & 0,46 & 0,93 & 0 & 9 & 45,29 & 0 \\
\hline 3 & & 0,93 & 1,39 & 0 & 34 & 50,08 & 0 \\
\hline 4 & & 1,39 & 1,86 & 7 & 46 & 61,29 & 16,33 \\
\hline 5 & & 1,86 & 2,32 & 8 & 31 & 72,41 & 26,86 \\
\hline 6 & & 2,32 & 2,79 & 0 & 4 & 66,53 & 0 \\
\hline 7 & & 2,79 & 3,25 & 0 & 1 & 59,06 & 0 \\
\hline
\end{tabular}

(1) Wadt et al. (1998). ${ }^{(2)}$ LIi: limite inferior da classe "i”. ${ }^{(3)}$ LSi: limite superior da classe "i”. ${ }^{(4)}$ P1: produtividade média dos talhões de alta produtividade na classe "i”. ${ }^{(5)} \mathrm{P} 2$ : frequência de talhões de alta produtividade na classe "i”, em relação ao total de talhões de alta produtividade. ${ }^{(6)}$ PRODi: frequência de talhões de alta produtividade, em relação ao total de talhões na classe "i". 
Quadro 2. Faixa normal (FN) de macro e micronutrientes em folha de cana-de-açúcar obtida pelos métodos ChM(1), DRIS(2,3) e CND(4,5) e nível crítico (NC)(6) determinado pelo critério de distribuição normal reduzida para cana-de-açúcar, em 1996-1997

\begin{tabular}{|c|c|c|c|c|c|c|c|c|c|c|}
\hline \multirow{2}{*}{ Nutriente } & \multicolumn{2}{|c|}{ ChM } & \multicolumn{2}{|c|}{ DRIS } & \multicolumn{2}{|c|}{ CND } & \multicolumn{2}{|c|}{ Malavolta et al. (1997) } & \multicolumn{2}{|c|}{ Raij et al. (1996) } \\
\hline & FN & NC & FN & NC & FN & NC & FN & $\mathrm{NC}$ & FN & $\mathrm{NC}$ \\
\hline & & & & & $-\mathrm{g} \mathrm{kg}^{-1}$ & & & & & \\
\hline $\mathrm{N}$ & $13,0-15,0$ & 14,5 & $12,6-15,2$ & & $12,6-16,5$ & & $19,0-21,0$ & & $18,0-25,0$ & \\
\hline $\mathrm{P}$ & $2,5-2,8$ & 1,8 & $1,8-2,3$ & & $1,5-2,2$ & & $2,0-2,4$ & & $1,5-3,0$ & \\
\hline $\mathrm{K}$ & $11,2-14,3$ & 9,9 & $9,6-12,5$ & & $7,6-12,0$ & & $11,0-13,0$ & & $10,0-16,0$ & \\
\hline $\mathrm{Ca}$ & $2,6-3,6$ & 4,0 & $2,6-3,6$ & & $3,3-4,8$ & & $8,0-10,0$ & & $2,0-8,0$ & \\
\hline $\mathrm{Mg}$ & $3,7-4,3$ & 2,4 & $2,0-3,0$ & & $1,6-3,2$ & & $2,0-3,0$ & & $1,0-3,0$ & \\
\hline $\mathrm{S}$ & $1,9-2,3$ & 1,6 & $1,5-1,9$ & & $1,3-1,8$ & & $2,0-3,0$ & & $1,5-3,0$ & \\
\hline $\mathrm{Cu}$ & $4,6-6,6$ & 4,8 & $3,8-5,0$ & & $3,8-5,6$ & & $8,0-10,0$ & & $6,0-15,0$ & \\
\hline $\mathrm{Mn}$ & $56,7-73,0$ & 79,5 & $55,9-78,3$ & & $52,7-86,3$ & & $100,0-250,0$ & & $25,0-250,0$ & \\
\hline $\mathrm{Zn}$ & $24,8-27,9$ & 12,9 & $12,6-14,8$ & & $13,0-16,4$ & & $25,0-50,0$ & & $10,0-50,0$ & \\
\hline
\end{tabular}

(1) Faixa normal estimada a partir dos limites superior e inferior das classes de frequência com maiores valores de chance matemática (Wadt et al., 1998). ${ }^{(2)}$ Macronutrientes: Para um índice DRIS calculado por Beaulfils (1971), determinado pela concentração do nutriente com índice igual a zero $\pm 2 / 3$, de acordo com Urano et al. (2007). ${ }^{(3)}$ Micronutrientes: Para um índice DRIS calculado por Beaufils (1971), determinado pela concentração do nutriente com índice igual a zero $\pm 2 / 3$, de acordo com Kuhiraha (2004). ${ }^{(4)}$ Macronutrientes: Para um índice CND calculado conforme Khiari et al. (2001) e determinado pela concentração do nutriente com índice igual a zero $\pm 2 / 3$, de acordo com Urano et al. (2007). ${ }^{(5)}$ Micronutrientes: Para um índice CND calculado conforme Khiari et al. (2001) e determinado pela concentração do nutriente com índice igual a zero $\pm 2 / 3$, de acordo com Kuhiraha (2004). ${ }^{(6)}$ Nível crítico determinado pelo critério da distribuição normal reduzida, conforme Maia et al. (2001).

Quadro 3. Valores de chances matemáticas (ChM) estabelecidos para diferentes classes de distribuição de teores dos micronutrientes em amostras de folhas de cana-de-açúcar coletadas em uma lavoura comercial, em 1996-1997

\begin{tabular}{|c|c|c|c|c|c|c|c|}
\hline Classe & Nutriente & $\mathbf{L I i}^{(2)}$ & $\mathbf{L S i}^{(3)}$ & $P 1^{(4)}$ & $\mathrm{P} 2^{(5)}$ & PRODi $^{(6)}$ & ChM \\
\hline & & \multicolumn{2}{|c|}{$\mathrm{mg} \mathrm{kg}^{-1}$} & & & $\%$ & $\mathrm{tha}^{-1}$ \\
\hline 1 & $\mathrm{Cu}$ & 2,68 & 4,62 & 6 & 57 & 53,07 & 10,89 \\
\hline 2 & & 4,62 & 6,56 & 9 & 65 & 65,30 & 18,81 \\
\hline 3 & & 6,56 & 8,50 & 0 & 2 & 72,09 & 0 \\
\hline 4 & & 8,50 & 10,44 & 0 & 2 & 71,52 & 0 \\
\hline 1 & $\mathrm{Mn}$ & 24,18 & 40,45 & 0 & 9 & 59,44 & 0 \\
\hline 2 & & 40,45 & 56,72 & 3 & 26 & 60,49 & 9,19 \\
\hline 3 & & 56,72 & 72,99 & 6 & 22 & 68,56 & 22,64 \\
\hline 4 & & 72,99 & 89,27 & 3 & 27 & 59,29 & 8,83 \\
\hline 5 & & 89,27 & 105,54 & 3 & 18 & 60,65 & 11,07 \\
\hline 6 & & 105,54 & 121,81 & 0 & 13 & 46,87 & 0 \\
\hline 7 & & 121,81 & 138,09 & 0 & 5 & 50,52 & 0 \\
\hline 8 & & 138,09 & 154,36 & 0 & 3 & 38,80 & 0 \\
\hline 9 & & 154,36 & 170,63 & 0 & 2 & 49,21 & 0 \\
\hline 10 & & 170,63 & 186,91 & 0 & 1 & 59,06 & 0 \\
\hline 1 & $\mathrm{Zn}$ & 6,19 & 9,29 & 3 & 22 & 55,57 & 9,17 \\
\hline 2 & & 9,29 & 12,40 & 5 & 38 & 62,24 & 13,03 \\
\hline 3 & & 12,40 & 15,51 & 2 & 40 & 56,23 & 4,59 \\
\hline 4 & & 15,51 & 18,61 & 4 & 18 & 67,70 & 16,48 \\
\hline 5 & & 18,61 & 21,72 & 0 & 6 & 56,88 & 0 \\
\hline 6 & & 21,72 & 24,83 & 0 & 1 & 74,04 & 0 \\
\hline 7 & & 24,83 & 27,94 & 1 & 1 & 84,14 & 21,72 \\
\hline
\end{tabular}

(1) Wadt et al. (1998). ${ }^{(2)}$ LIi: limite inferior da classe "i". ${ }^{(3)}$ LSi: limite superior da classe "i". ${ }^{(4)}$ P1: produtividade média dos talhões de alta produtividade na classe "i". (5) P2: frequência de talhões de alta produtividade na classe "i”, em relação ao total de talhões de alta produtividade. ${ }^{(6)}$ PRODi: Frequência de talhões de alta produtividade, em relação ao total de talhões na classe "i". 
Quadro 4. Modelos estatísticos dos relacionamentos entre teores de nutrientes e índices DRIS e CND em amostras de folhas de cana-de-açúcar coletadas em uma lavoura comercial, em 19961997

\begin{tabular}{|c|c|c|c|}
\hline Nutriente & $\begin{array}{c}\text { Método } \\
\text { estatístico }\end{array}$ & Modelo & $\mathbf{R}^{2}$ \\
\hline \multirow{2}{*}{$\mathrm{N}$} & DRIS & $N=12,88-0,44 x$ & 0,53 \\
\hline & CND & $\mathrm{N}=14,15+1,39 \mathrm{x}$ & 0,63 \\
\hline \multirow{2}{*}{$\mathrm{P}$} & DRIS & $P=2,06+0,004 x$ & 0,64 \\
\hline & CND & $P=2,06+0,41 x$ & 0,78 \\
\hline \multirow{2}{*}{$\mathrm{K}$} & DRIS & $K=11,05+0,02 x$ & 0,60 \\
\hline & CND & $\mathrm{K}=11,22+2,17 \mathrm{x}$ & 0,66 \\
\hline \multirow{2}{*}{$\mathrm{Ca}$} & DRIS & $\mathrm{Ca}=3,13+0,001 \mathrm{x}$ & 0,82 \\
\hline & CND & $\mathrm{Ca}=3,25+0,93 \mathrm{x}$ & 0,83 \\
\hline \multirow{2}{*}{$\mathrm{Mg}$} & DRIS & $\mathrm{Mg}=2,25+0,007 \mathrm{x}$ & 0,72 \\
\hline & CND & $\mathrm{Mg}=2,43+0,77 \mathrm{x}$ & 0,83 \\
\hline \multirow[t]{2}{*}{$\mathrm{S}$} & DRIS & $\mathrm{S}=1,71+0,002 \mathrm{x}$ & 0,53 \\
\hline & CND & $\mathrm{S}=1,75+0,16 \mathrm{x}$ & 0,76 \\
\hline \multirow{2}{*}{$\mathrm{Cu}$} & DRIS & $\mathrm{Cu}=4,42+0,008 \mathrm{x}$ & 0,70 \\
\hline & CND & $\mathrm{Cu}=4,62+0,87 \mathrm{x}$ & 0,78 \\
\hline \multirow{2}{*}{$\mathrm{Mn}$} & DRIS & $\mathrm{Mn}=67,10+0,15 \mathrm{x}$ & 0,83 \\
\hline & CND & $\mathrm{Mn}=69,50+16,08 \mathrm{x}$ & 0,85 \\
\hline \multirow{2}{*}{$\mathrm{Zn}$} & DRIS & $\mathrm{Zn}=13,68+0,02 \mathrm{x}$ & 0,70 \\
\hline & CND & $\mathrm{Zn}=14,73+1,45 \mathrm{x}$ & 0,97 \\
\hline
\end{tabular}

estatísticos entre os teores de nutrientes nas folhas de cana-de-açúcar e os índices CND, sendo considerado na avaliação o modelo linear (Quadro 4).

As faixas normais de nutrientes determinadas pelos métodos DRIS e CND foram semelhantes, porém diferenciaram-se dos resultados obtidos pelo método ChM, concordando com resultados encontrados por Serra et al. (2010) e Camacho et al. (2012a).

A partir do trabalho de Reis Junior \& Monerat (2003), foi demonstrada uma relação positiva e significativa entre teores foliares e índices DRIS em cana-de-açúcar, sendo encontrados valores semelhantes neste trabalho. Como os demais métodos apresentam resultados semelhantes, pode-se afirmar que houve correlação positiva para os três métodos matemáticos de diagnose foliar.

As faixas determinadas por Malavolta et al. (1997) ficaram muito distantes da faixa de suficiência encontrada pelos três métodos utilizados neste trabalho, exceto para $\mathrm{P}, \mathrm{K}, \mathrm{Mg}$.

As faixas normais encontradas para $\mathrm{N} \mathrm{e} \mathrm{Cu}$ foram análogas às normais discriminadas por Raij et al. (1996); contudo, os valores das faixas normais para os demais nutrientes foram semelhantes e estiveram dentro da faixa de suficiência recomendada por Raij et al. (1996), possuindo menor amplitude e com limites superiores muito baixos.
Trabalhos realizados por Urano et al. (2007), Serra et al. (2010) e Camacho et al. (2012a,b) também encontraram amplitudes de faixas normais muito aquém das encontradas na literatura, indicando que os métodos ChM, DRIS e CND, por serem desenvolvidos regionalmente, possuem maior especificidade. A menor amplitude dos valores de referências corrobora para maior eficiência na interpretação das análises, diminuindo a possibilidade de obtenção de baixas produtividades e com teores adequados de nutrientes na planta (Serra et al., 2010).

\section{CONCLUSÕES}

1. A utilização dos métodos propostos pode ser adotada para o diagnóstico nutricional e a calibração de teores ótimos para a cultura da cana-de-açúcar, a partir de monitoramento de lavouras comerciais.

2. As faixas normais obtidas pelos métodos em estudo apresentaram menor amplitude das classes de teores nutricionais, em relação às faixas de suficiência descritas pela literatura.

\section{AGRADECIMENTO}

Ao Sr. Roberto dos Anjos Reis Junior, por ceder os dados para este estudo.

\section{LITERATURA CITADA}

BEAUfILS, E.R. Physiological diagnosis - a guide for improving maize preproduction based principles developed for rubber trees. Fert. Soc. Sci. Afr. J., 1:130, 1971.

BEAUFILS, E.R. Diagnosis and recommendation integrated system (DRIS). Natal, University Natal, 1973. 130p. (Soil Science Bulletin.)

CAMACHO, M.A.; SILVEIRA, M.V.S.; CAMARGO, R.A. \& NATALE, W. Faixas normais de nutrientes pelos métodos ChM, DRIS e CND e nível crítico pelo método de distribuição normal reduzida para laranjeira-pera. R. Bras. Ci. Solo, 36:193-200, 2012a.

CAMACHO, M.A.; NATALE, W. \& BARBOSA, J.C. Faixa de suficiência para a cultura do algodão no centro-oeste do Brasil. II - Micronutrientes. Ci. Rural, 42:993-1000, 2012b.

COMPANHIA NACIONAL DE ABASTECIMENTO - CONAB. Acompanhamento da safra brasileira: Cana-de-açúcar, 2012/2013: Segundo levantamento. Brasília, 2012. Disponível em: <http://www.conab.gov.br>. Acesso em: 14 ago. de 2012.

CONOVER, N.J. Practical non parametric statistics. New York, John Wiley and Sons, 1971. 462p. 
HARTZ, T.K.; MIYAO, E.M. \& VALENCIA, J.G. DRIS evaluation of the nutritional status of processing tomato. Hortscience, 33:830-832, 1998.

JONES, W.W. Proposed modifications of the diagnosis and recommendation integrated system (DRIS) for interpreting plant analyses. Commun. Soil Sci. Plant Anal., 12:785-794, 1981.

KHIARI, L.; PARENT, L.E. \& TREMBLAY, N. Critical compositional nutrient indexes for sweet corn at early growth stage. Agron. J., 93:809-814, 2001.

KURIHARA, C.H. Demanda de nutrientes pela soja e diagnose de seu estado nutricional. Viçosa, MG, Universidade Federal de Viçosa, 2004. 101p. (Tese de Doutorado)

MAIA, C.E.; MORAIS, E.R.C. \& OLIVEIRA, M. Nível crítico pelo critério da distribuição normal reduzida: Uma nova proposta para interpretação de análise foliar. R. Bras. Eng. Agric. Amb., 5:235-238, 2001.

MALAVOLTA, E.; VITTI, G.C. \& OLIVEIRA, S.A. Avaliação do estado nutricional das plantas -princípios e aplicações. Piracicaba, Potafos, 1997. 319p.

MOURÃO FILHO, F.A.A.; AZEVEDO, J.C. \& NICK, J.A. Funções e ordem da razão dos nutrientes no estabelecimento de normas DRIS em laranjeira 'Valência'. Pesq. Agropec. Bras., 37:185-192, 2002.

OTTO, R.; VITTI, G.C. \& LUZ, P.H.C. Manejo da adubação potássica na cultura da cana-de-açúcar. R. Bras. Ci. Solo, 34:1137-1145, 2010.

PARENT, L.E.; CAMBOURIS, A.N. \& MUHAWENIMANA, A. Multivariate diagnosis of nutrient imbalance in potato crops. Soil Sci. Soc. Am. J., 58:1432-1438, 1994.

PARENT, L.E. \& DAFIR, M.A. Theoretical concept of compositional nutrient diagnosis. J. Am. Soc. Hortic. Sci., 117:239-242, 1992.
RAIJ, B.van; CANTARELlA, H.; QUAGGIO, J.A. \& FURLANI, A.M.C. Recomendações de adubação e calagem para o Estado de São Paulo. 2.ed. Campinas, Instituto Agronômico de Campinas, 1996. 285p.

REIS JUNIOR, R.A. \& MONNERAT, P.H. Diagnose nutricional da cana-de-açúcar em Campos dos Goytacazes, RJ. R. Bras. Ci. Solo, 26:367-372, 2002.

REIS JUNIOR, R.A. \& MONNERAT, P.H. DRIS norms validation for sugarcane crop. Pesq. Agropec. Bras., 38:379-385, 2003.

SERRA, A.P.; MARCHETTI, M.E.; VITORI NO, A.C.T.; NOVELINO, J.A. \& CAMACHO, M.A. Determinação de faixas normais de nutrientes no algodoeiro pelos métodos ChM, CND e DRIS. R. Bras. Ci. Solo, 34:97104, 2010.

URANO, E.O.M.; KURIHARA, C.H.; MAEDA, S. VITORINO, A.C.T.; GONÇALVES, M.C. \& MARCHETTI, M.E. Determinação de teores ótimos de nutrientes em soja pelos métodos chance matemática, sistema integrado de diagnose e recomendação e diagnose da composição nutricional. R. Bras. Ci. Solo, 31:63-72, 2007.

WADT, P.G.S.; ALVAREZ V., V.H.; NOVAIS, R.F.; FONSECA, S. \& BARROS, N.F. O método da Chance Matemática na interpretação de dados de levantamento nutricional de eucalipto. R. Bras. Ci. Solo, 22:773-778, 1998.

WADT, P.G.S.; DIAS, J.R.M.; PEREZ, D.V. \& LEMOS, C.O. Interpretação de índices DRIS para a cultura do cupuaçu. R. Bras. Ci. Solo, 36:125-135, 2012.

WALWORTH, J.L.; SUMNER, M.E.; ISAAC, R.A. \& PLANK, C.O. Preliminary DRIS norms for alfafa in the Southeastern United State and a comparison with the Midwest norms. Agron. J., 78:1046-1052, 1986. 